\title{
The Predictive Value of Pathologic Features in Pituitary Adenoma and Correlation with Pituitary Adenoma Recurrence
}

Jee Soon Kim • Youn Soo Lee Min Jung Jung ${ }^{1}$. Yong Kil Hong ${ }^{2}$

Department of Hospital Pathology, College of Medicine, The Catholic University

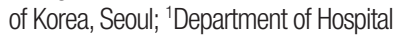
Pathology, Soonchunhyang University Hospital, Bucheon; '2Department of Neurosurgery, College of Medicine, The Catholic University of Korea, Seoul, Korea

Received: April 29, 2016

Revised: June 10, 2016

Accepted: June 30, 2016

\section{Corresponding Author}

Youn Soo Lee, MD, PhD

Department of Hospital Pathology, College of

Medicine, The Catholic University of Korea, 222

Banpo-daero, Seocho-gu, Seoul 06591, Korea

Tel: +82-2-2258-1626

Fax: +82-2-2258-1628

E-mail: lys9908@catholic.ac.kr

\begin{abstract}
Background: The 2004 World Health Organization classification introduced atypical pituitary adenoma (aPA), which was equivocally defined as invasion with increased mitotic activity that had a Ki-67 labeling index (LI) greater than 3\%, and extensive p53 immunoreactivity. However, aPAs that exhibit all of these features are rare and the predictive value for recurrence in pituitary adenomas (PAs) remains uncertain. Thus, we sought to characterize pathological features of PAs that correlated with recurrence. Methods: One hundred and sixty-seven cases of surgically resected PA or aPA were retrieved from 2011 to 2013 in Seoul St. Mary's Hospital. Among them, 28 cases were confirmed to be recurrent, based on pathologic or radiologic examination. The pathologic characteristics including mitosis, invasion, Ki-67 LI and p53 immunoreactivity were analyzed in relation to recurrence. Results: Analysis of the pathologic features indicated that only Ki-67 LI over $3 \%$ was significantly associated with tumor recurrence $(p=.02)$. The cases with at least one pathologic feature showed significantly higher recurrence rates $(p<.01)$. Analysis indicated that cases with two pathologic features, Ki-67 LI over 3\% and extensive p53 immunoreactivity $20 \%$ or more, were significantly associated with tumor recurrence $(p<.01)$. Conclusions: Based on these results, PA tumor recurrence can be predicted by using mitosis, invasion, Ki-67 LI (3\%), or extensive p53 immunoreactivity $(\geq 20 \%)$. Assessment of these features is recommended for PA diagnosis for more accurate prediction of recurrence.
\end{abstract}

Key Words: Pituitary neoplasms; Recurrence; Ki-67 antigen; Tumor suppressor protein p53
Pituitary adenomas (PAs) are defined as adenomas that are derived from adenohypophyseal cells in the sella turcica. ${ }^{1}$ PAs account for more than $10 \%$ of all intracranial tumors ${ }^{2}$ and are relatively common. ${ }^{3,4}$ Clinically, PAs are classified into nonfunctioning and functioning. They are divided into groups based on size, which include microadenomas $(<1 \mathrm{~cm})$, macroadenomas $(\geq$ $1 \mathrm{~cm}$ and $<4 \mathrm{~cm}$ ), and giant adenomas $(\geq 4 \mathrm{~cm})$. Based on pathology, classification in the past was divided into three types (acidophilic, basophilic, and chromophobic), which was based only on morphologic features. However, they have recently been categorized into more fragmented classifications. ${ }^{1,5}$ The following characteristics are associated with clinically aggressive behavior: densely granulated prolactin-secreting adenomas, sparsely granulated monohormonal growth hormone-secreting adenoma, acidophilic stem cells, plurihormonal growth hormonesecreting adenoma, Crooke's cell adenoma (aggressive variant of adrenocorticotropic hormone-secreting adenoma), sparsely granulated adrenocorticotropic hormone-secreting adenoma, thyroid-stimulating hormone-secreting adenoma, Null-cell adenoma (oncocytoma), and silent subtype 3 tumor. ${ }^{6,7}$ Evaluation of prognosis is limited, due to a discrepancy between immunohistochemically detected (produced) and secreted hormones.

Apart from the subtypes above, invasion into sinuses or recurrence after follow-up have often been identified by radiologic imaging techniques, such as magnetic resonance imaging. In 2004, the World Health Organization (WHO) designated a new group of atypical pituitary adenomas (aPA; International Classification of Diseases for Oncology [ICD-O] code 1). The aPA is defined by some markers that reflect the aggressive potential of PA. According to the $\mathrm{WHO}, \mathrm{aPA}$ is a PA with aggressive features, such as invasion, and the diagnostic criteria include increased mitotic activity, Ki-67 labeling index (LI) $>3 \%$, and extensive p53 immunoreactivity. ${ }^{1}$ Pituitary carcinomas (PC) with poor prognosis (4-year mean survival) can be diagnosed when craniospinal or systemic metastasis is confirmed. ${ }^{8}$ The aggressive features, however, are not well-defined and can have different interpretations, depending on the individual investigators. ${ }^{5,9}$ For example, Zada et al. ${ }^{9}$ excluded invasion from the criteria and 
reported $14.8 \%$ as a prevalence of aPA. However, when Saeger et al. ${ }^{5}$ applied criteria, including invasion, they reported a prevalence of $2.7 \%$. In daily practice, the aPAs that correspond to all four features are actually very rare; therefore, the predictive value of these criteria for recurrence is questionable. Moreover, a recurrence has been reported in up to $36 \%$ of typical PAs. ${ }^{8-14}$ Aside from Ki-67 LI, definite cut-offs for increased mitotic activity and extensive p 53 immunoreactivity have not been described in detail. $^{1}$

The purpose of this study was to investigate the association between current aPA diagnostic criteria and tumor recurrence and to characterize the pathological features of PAs in order to establish more accurate prediction of tumor recurrence.

\section{MATERIALS AND METHODS}

The Institutional Review Board of the Catholic Medical Center approved this study (IRB No. KC14S1SI0833).

From 2011 to 2013 in Seoul St. Mary's Hospital, surgicallyexcised endocrine pituitary tumors were reviewed by two pathologists. One hundred and sixty-seven cases of pituitary tumors that were sequentially enrolled were identified. Only one aPA was included in these cases. Histologic features, such as increased mitotic activity and microscopic invasion (dura, bone, sinus, etc.), were assessed in relation to recurrence. The recurrence was defined as a newly-found mass that was diagnosed pathologically or identified radiologically (in the group without remnant tumors after gross total removal) or a mass that was larger than the first diagnosed mass size, detected by radiologic assessment after follow-up (in the group with remnant tumors). In the group without remnant tumors, after gross total removal, recurrence was defined as a newly-found mass that was pathologically diagnosed or radiologically identified. Immunohistochemistry analysis was performed with Ki-67 (ready-to-use, Roche, Indianapolis, IN, USA) and p53 (ready-to-use, Roche). Clinical data review was used to determine age, gender, residual mass, tumor size $(<$ $1 \mathrm{~cm}$, microadenoma; $\geq 1 \mathrm{~cm}$ and $<4 \mathrm{~cm}$, macroadenoma; $\geq 4$ $\mathrm{cm}$, giant adenoma) and recurrence after operation.

The following criteria were used to define aPA: (1) invasion, (2) a Ki-67 LI greater than 3\%, (3) extensive p53 immunoreactivity, and (4) increased mitotic activity. PC were diagnosed by identifying distant metastases. ${ }^{8}$ Invasion into adjacent dura, bone and/or surrounding anatomical structures were taken into account. We manually counted 1,000 cells in the hot spot area for Ki-67 LI. Extensive p53 immunoreactivity was defined as intensive nuclear reactivity of $20 \%$ or more in tumor cells and increased mitotic activity was defined as 2/10 high power field (HPF) or more. The four features and cut-offs listed above were analyzed in relation to recurrence, in combination with or without other characteristics as follows: invasion, Ki-67 LI > 3\%, extensive p53 immunoreactivity, mitosis $\geq 2 / 10 \mathrm{HPF}$, and any additional criteria, as listed above. Other characteristics such as gender, age at diagnosis, tumor size, presence of residual tumor and recurrence were also analyzed. For the qualitative variable comparison, student $\mathrm{t}$ test, Pearson's chi-square test and linear by linear association were used. Every p-value used in this study was two-sided, and a value less than $.05(\mathrm{p}<.05)$ was counted as statistically significant. Moreover, odds ratios (OR) were calculated. OR were used to measure the association between a variable and an outcome. The OR is defined as the probability of an outcome occurring with a specific variable, compared to the odds that the outcome will occur without that variable. ${ }^{15}$ SPSS

Table 1. Clinical characteristics and recurrence rates

\begin{tabular}{|c|c|c|c|c|c|}
\hline Characteristic & All patients & Patients with recurrence & Patients without recurrence & $\mathrm{p}$-value & Odds ratio \\
\hline No. (\%) & $167(100)$ & $28(16.8)$ & $139(83.2)$ & & \\
\hline Mean age (range) & $49(19-80)$ & $43(20-71)$ & $50(19-80)$ & $.02^{*}$ & - \\
\hline Male & $79(47.3)$ & $12(15.2)$ & $67(84.8)$ & & \\
\hline Female & $88(52.7)$ & $16(18.2)$ & $72(81.8)$ & & \\
\hline Tumor size ${ }^{a}$ & & & & $>.05$ & - \\
\hline Microadenoma & $15(9)$ & 2 (13.3) & $13(86.7)$ & & \\
\hline Macroadenoma & $141(84.4)$ & $21(14.9)$ & $120(85.1)$ & & \\
\hline Giant adenoma & $11(6.6)$ & $5(45.5)$ & $6(54.5)$ & $<.01^{*}$ & 4.85 \\
\hline Residual mass & & & & $<.01^{*}$ & 6.27 \\
\hline Absent & $118(70.7)$ & $10(8.5)$ & $105(89)$ & & \\
\hline Present & 49 (29.3) & $18(36.7)$ & 31 (63.3) & & \\
\hline
\end{tabular}

Values are presented as number (\%).

*Significant difference $(p<.05)$.

${ }^{a}$ Classified into microadenoma $(<1 \mathrm{~cm})$, macroadenoma $(\geq 1 \mathrm{~cm}$ and $<4 \mathrm{~cm})$, giant adenoma $(\geq 4 \mathrm{~cm})$. 
statistical software ver. 21.0 (IBM Co., Armonk, NY, USA) was used for statistical analyses.

\section{RESULTS}

The clinical characteristics and recurrence rates are summarized in Table 1. Twenty-eight out of 167 patients (16.8\%) experienced recurrence. The mean age at first diagnosis in the group with recurrence was 43 years which was significantly younger than that of the group without recurrence, which was 50 years $(\mathrm{p}=$ .02). Although there were more female patients than male patients, the difference was not statistically significant $(\mathrm{p}=.61)$. Recurrence rates were $13.3 \%$ in microadenoma, $14.9 \%$ in macroadenoma, and $45.5 \%$ in giant adenoma. The analysis indicated that a higher recurrence rate was associated with larger tumor size, although this result was not statistically significant ( $\mathrm{p}>.05)$. Analysis of the giant adenomas in relation to recurrence indicated that there was a significant association with recurrence $(\mathrm{p}<.01$; $\mathrm{OR}, 4.85)$. The presence of residual mass after surgery was observed in 49 patients (29.3\%). In the group with residual mass, 18 patients (36.7\%) experienced recurrence, which was significantly higher than recurrence (8.5\%) in the group without residual mass ( $\mathrm{p}<.01 ; \mathrm{OR}, 6.27)$.

Reclassification of the study population using histopathologic features with recurrence rates are summarized in Tables $2-4$. In the group with recurrence, the mean $\mathrm{Ki}-67 \mathrm{LI}$ was $4.04 \%$ and was greater than that of the group without recurrence, which was $1.95 \%$; however, this result was not significantly different $(\mathrm{p}=.14)$. The Ki-67 LI over $3 \%$ was significantly associated with recurrence ( $\mathrm{p}=.02 ; \mathrm{OR}, 2.99)$. In regard to invasion, the

Table 2. Reclassification of the study population using histopathologic criteria

\begin{tabular}{|c|c|c|c|c|c|c|}
\hline \multirow{2}{*}{ Characteristic } & \multicolumn{2}{|c|}{ Patients with recurrence $(n=28)$} & \multicolumn{2}{|c|}{ Patients without recurrence $(n=139)$} & \multirow{2}{*}{$p$-value } & \multirow{2}{*}{ Odds ratio } \\
\hline & Present & Absent & Present & Absent & & \\
\hline Ki-67, mean & \multicolumn{2}{|c|}{4.04} & \multicolumn{2}{|c|}{1.95} & .14 & \\
\hline Labeling index (>3\%) & $9(32.1)$ & 19 (67.9) & $19(13.7)$ & $120(86.3)$ & $.02^{*}$ & 2.99 \\
\hline Invasion & $9(32.1)$ & $19(67.9)$ & $27(19.4)$ & $112(80.6)$ & .14 & \\
\hline p53 immunoreactivity ( $\geq 20 \%)$ & $2(7.1)$ & $26(92.9)$ & $3(2.2)$ & $136(97.8)$ & .16 & \\
\hline Mitosis, mean & \multicolumn{2}{|c|}{1.11} & \multicolumn{2}{|c|}{.26} & .31 & \\
\hline$\geq 2 / 10 \mathrm{HPF}$ & $3(10.7)$ & 25 (89.3) & $4(2.9)$ & $135(97.1)$ & .06 & \\
\hline
\end{tabular}

Values are presented as number (\%).

*Significant difference $(p<.05)$.

Table 3. Reclassification of the study population using histopathologic criteria: cases with at least any one or two or all of the four criteria in relation to recurrence

\begin{tabular}{|c|c|c|c|c|c|c|}
\hline \multirow{2}{*}{ Combination of criteria } & \multicolumn{2}{|c|}{ Patients with recurrence $(n=28)$} & \multicolumn{2}{|c|}{ Patients without recurrence $(n=139)$} & \multirow{2}{*}{ p-value } & \multirow{2}{*}{ Odds ratio } \\
\hline & Present & Absent & Present & Absent & & \\
\hline \multicolumn{7}{|l|}{ I/K3/p20/M2 } \\
\hline$\geq 1^{\mathrm{a}}$ & $17(60.7)$ & $11(39.3)$ & $39(28.1)$ & $100(71.9)$ & $<.01^{*}$ & 3.96 \\
\hline$\geq 2^{b}$ & $4(14.3)$ & $24(85.7)$ & $14(10.1)$ & $125(89.9)$ & .51 & \\
\hline All $(4)^{c}$ & $1(3.6)$ & $27(96.4)$ & 0 & $139(100)$ & $.03^{*}$ & \\
\hline
\end{tabular}

I, invasion; K3, Ki-67 LI > 3\%; p20, p53 immunoreactivity $\geq 20 \%$; M2, mitosis $\geq 2 / 10 \mathrm{HPF}$.

*Significant difference $(p<.05)$.

aSatisfying any one or more criteria on the left; 'Satisfying any two or more criteria on the left; 'Satisfying all of the criteria on the left.

Table 4. Reclassification of the study population using histopathologic criteria (3): cases with two criteria among four criteria in relation to recurrence

\begin{tabular}{|c|c|c|c|c|c|}
\hline \multirow{2}{*}{ Combination of criteria } & \multicolumn{2}{|c|}{ Patients with recurrence $(n=28)$} & \multicolumn{2}{|c|}{ Patients without recurrence $(n=139)$} & \multirow{2}{*}{$p$-value } \\
\hline & Present & Absent & Present & Absent & \\
\hline $\mathrm{K} 3$ and I & $3(10.7)$ & $25(89.3)$ & $9(6.5)$ & $130(93.5)$ & .43 \\
\hline $\mathrm{K} 3$ and $\mathrm{p} 20$ & $2(7.1)$ & $26(92.9)$ & 0 & $139(100)$ & $<.01^{*}$ \\
\hline $\mathrm{K} 3$ and $\mathrm{M} 2$ & $1(3.6)$ & $27(96.4)$ & $2(1.4)$ & $137(98.6)$ & .44 \\
\hline I and p20 & $1(3.6)$ & $27(96.4)$ & $2(1.4)$ & $137(98.6)$ & .44 \\
\hline I and $\mathrm{M} 2$ & $1(3.6)$ & $27(96.4)$ & $1(.7)$ & $138(99.3)$ & .21 \\
\hline $\mathrm{p} 20$ and $\mathrm{M} 2$ & $1(3.6)$ & $27(96.4)$ & 0 & $139(100)$ & $.03^{*}$ \\
\hline
\end{tabular}

K3, Ki-67 LI > 3\%; l, invasion; p20, p53 immunoreactivity $\geq 20 \%$; M2, mitosis $\geq 2 / 10 H P F$.

*Significant difference $(p<.05)$. 
recurrence rate was slightly higher in cases with invasion (32.1\%) than in cases without invasion (19.4\%); however, this result was not significant $(\mathrm{p}=.14)$. Only five patients showed extensive p53 immunoreactivity and two of them experienced recurrence $(\mathrm{p}=.16)$. We assessed the relationship between increased mitotic activity and recurrence but did not identify any significant differences, and in most cases, mitosis with 1/10HPF or less was observed. The mean mitotic count was $1.11 / 10 \mathrm{HPF}$ in the group with recurrence versus $0.26 / 10 \mathrm{HPF}$ in the group without recurrence $(\mathrm{p}=.31)$ (Table 2$)$. There was a significant correlation between the group that satisfied at least one of the four identified features and recurrence $(\mathrm{p}<.01 ; \mathrm{OR}, 3.96)$. The cases with two or more features were not significantly correlated with the results $(\mathrm{p}=.51)$ (Table 3). Among the cases with two specific features, the group with $\mathrm{Ki}-67 \mathrm{LI}>3 \%$ and extensive p53 immunoreactivity experienced statistically significant recurrence $(\mathrm{p}<.01)$. The group with extensive p53 immunoreactivity and increased mitotic activity had significant recurrence; however, only one of these cases was identified $(\mathrm{p}=.03)$. The other relationships were analyzed; however, no statistically significant relationship was identified (Table 4). Only one aPA patient met all four of the WHO criteria (Fig. 1), and the only case which satisfied any 3 or 4 of the criteria was determined to have a statistically significant relationship with recurrence $(\mathrm{p}=.03$ ) (Table 3). Approximately 1 year from the first diagnosis, this case was confirmed be PC after the metastasis was identified to the distant cerebrum and skull (Fig. 2).

\section{DISCUSSION}

In this present study, analyzing cases with at least one of the diagnostic criteria contributed to predicting recurrence. This result indicates that recurrence would be significantly lower in patients that do not show any of the aPA criteria, compared
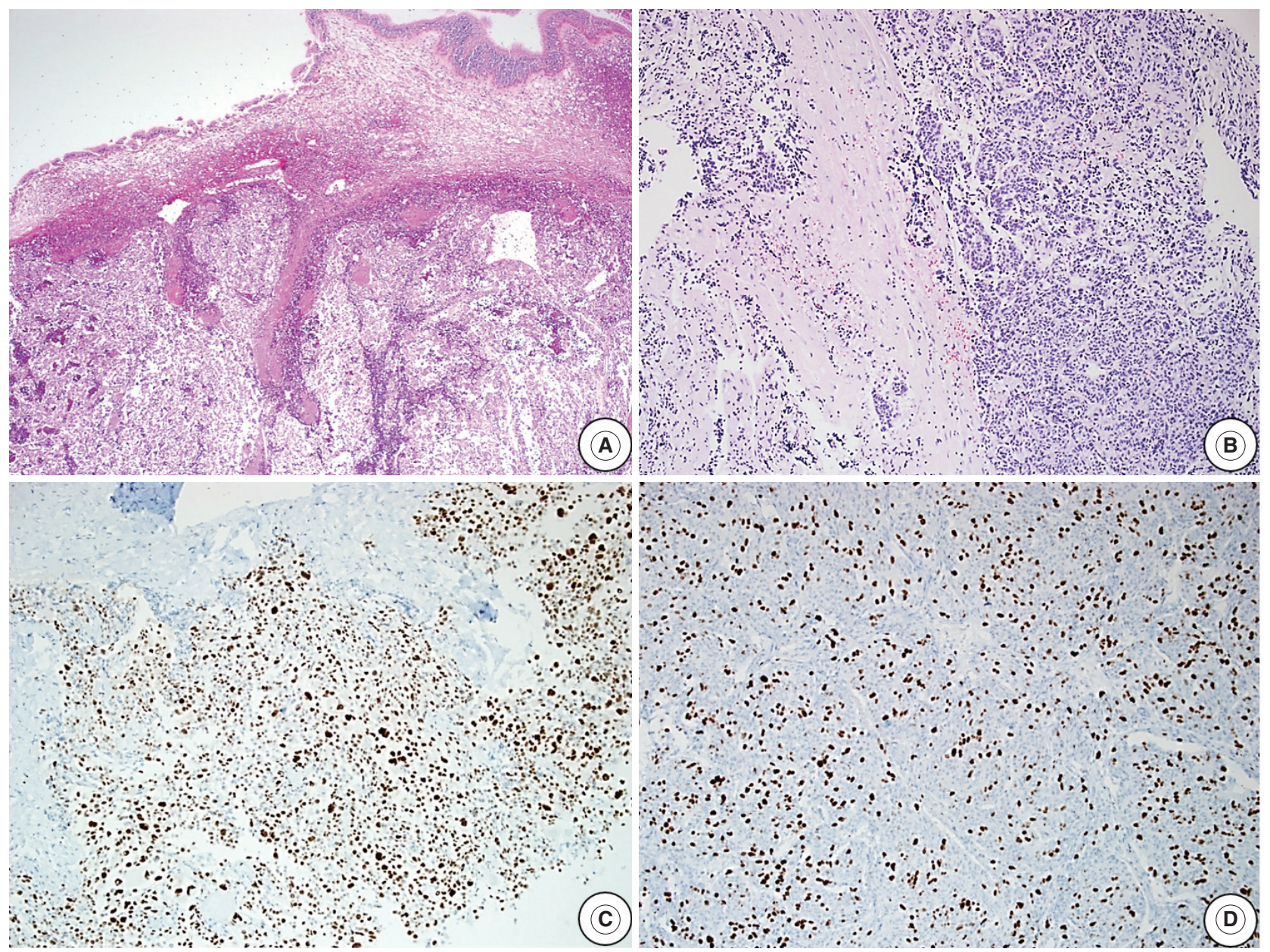

Fig. 1. Microscopic findings of an atypical pituitary adenoma case with sphenoid sinus and dura invasion. (A) Tumor cells invading the sphenoid sinus. (B) Foci of tumor cell infiltration into the dura. (C) Tumor cells show extensive p53 immunoreactivity. (D) High Ki-67 labeling index (about $40 \%)$ is noted. 

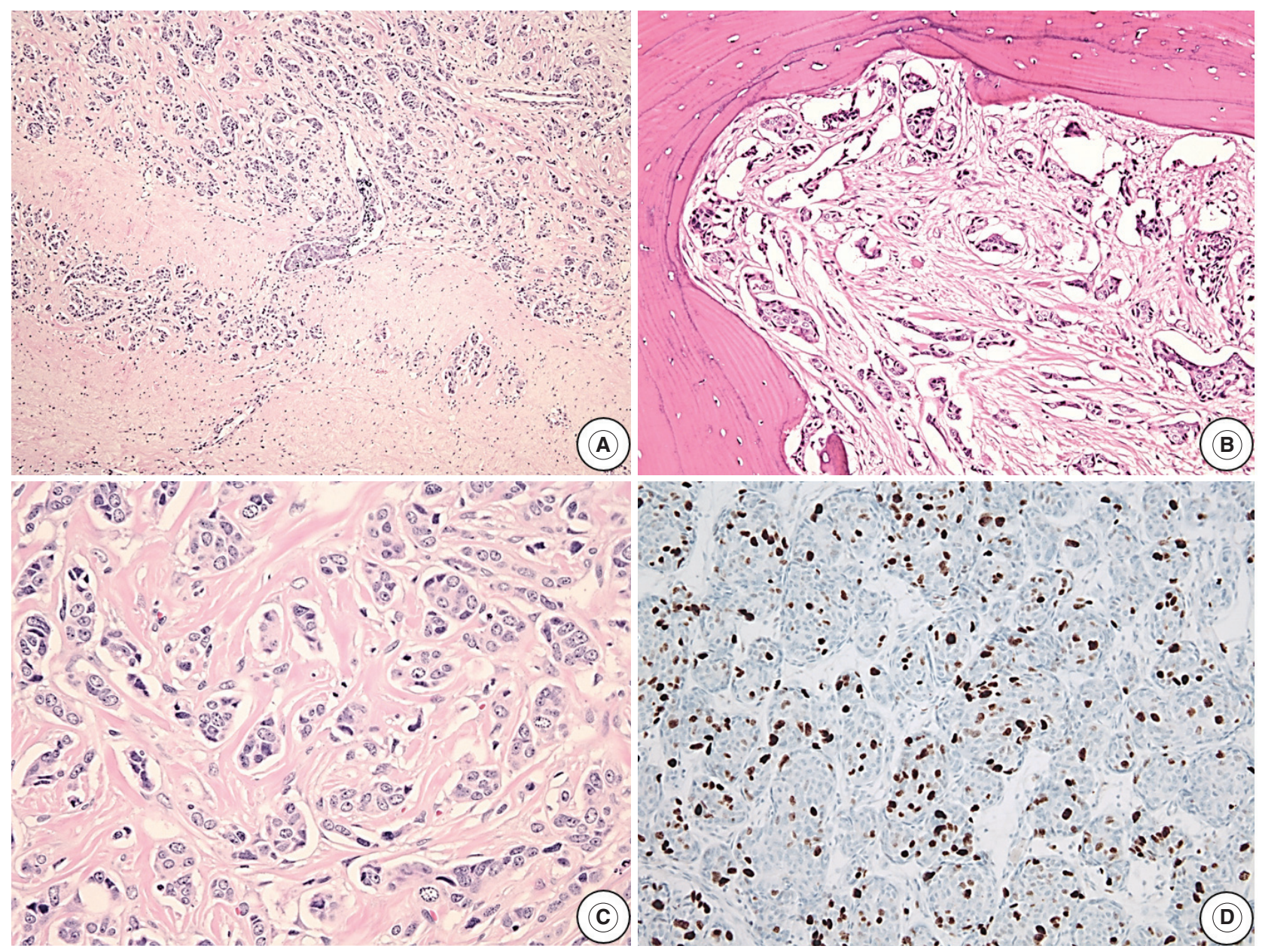

Fig. 2. Microscopic findings of a pituitary carcinoma case with metastasis to the distant cerebrum and skull. (A) The tumor cells metastasize to the cerebrum. (B) The skull bone tissue is infiltrated by the metastatic tumor cells. (C) Infiltrating tumor cells with mitotic activity. (D) High Ki-67 labeling index (about 15\%) is observed.

with other patients. Among the four criteria, cases with Ki-67 $\mathrm{LI}>3 \%$ alone or along with extensive p53 immunoreactivity showed similar results.

Although many predictive factors have been reported to be related with tumor recurrence in PAs, there is controversy about whether these factors are practically useful. The WHO's diagnostic criteria for aPA is poorly defined and its application is currently under discussion. ${ }^{9,16-20}$ There is no definite cut-off for extensive p53 immunoreactivity and increased mitotic activity. The features in the criteria are also frequently observed in PA. We found that a valid cut-off for KI-67 LI is $>3 \%$. Matsuyama ${ }^{21}$ and Lee et al..$^{10}$ compared $\mathrm{Ki}-67$ LI between patients with and without recurrence and found that it was significantly different. However, Sadeghipour et al. ${ }^{11}$ and Hadzhiyanev et al. ${ }^{12}$ found no significant result when they compared mean Ki-67 LI between patients with recurrence and those without recurrence. Many studies have compared PA and aPA. Miermeister et al. ${ }^{22}$ defined the inclusion criteria of aPA as a minimum three of criteria and reported $\mathrm{Ki}-67 \mathrm{LI}>4 \%$ as the best marker to discriminate between PA and aPA. In our study, however, Ki-67 LI > 4\% showed no significant difference in relation to recurrence $(\mathrm{p}=$ .28). We analyzed $\mathrm{Ki}-67 \mathrm{LI}$ in the group with recurrence, and the mean value was 4.04 and the standard deviation was 7.24. Five cases with $\mathrm{Ki}-67 \mathrm{LI}>3 \%$ but $\leq 4 \%$ were found in the group with recurrence $(n=28)$.

We concluded that invasion alone was not a significant factor for predicting recurrence. Similarly, Matsuyama ${ }^{21}$ found that suprasellar extension and/or cavernous sinus invasion were not meaningfully associated with recurrence. Several studies comparing PA and aPA showed significant results. ${ }^{9,13,14,22}$ Specifically, Chiloiro et al. ${ }^{14}$ reported that the cases with cavernous invasion were associated with significant results; however, the cases with the other characteristics, including suprasellar extension, were not significant. Our results could be limited because we did not 
classify cases by the location of the invasion.

In this study, increased mitotic activity was not associated with recurrence. Miermeister et al. ${ }^{22}$ suggested the cut-off value of mitotic count $2 / 10 \mathrm{HPF}$ or more as the best criterion to discriminate between PA and aPA. When applying the criterion in this study, there was no significant relationship between recurrence and increased mitotic activity. Moreover, when comparing the mean mitotic count between the groups with and without recurrence, there was no significant difference. In comparison, Lee et al. ${ }^{10}$ suggested the cutoff value of mitotic count $1 / 10 \mathrm{HPF}$ or more as a good predictive marker for recurrence. We also applied this criterion in our study, although there was no meaningful association between increased mitosis (1/10HPF or more) and high recurrence rate (data not shown).

Analysis of extensive p53 immunoreactivity did not indicate any significant results. Similarly, Hadzhiyanev et al. ${ }^{12}$ compared p53 nuclear positivity (at least weak or focal expression) and did not identify any significant difference. However, Lee et al. ${ }^{10}$ compared $\mathrm{p} 53$ intensive nuclear activity $\geq 3 \%$ between patients with and without recurrence and reported significant results. Miermeister et al. ${ }^{22}$ suggested p53 intensive nuclear activity $\geq 2 \%$ as the best criterion for aPA.

Cases with $\mathrm{Ki}-67 \mathrm{LI}>3 \%$ along with extensive p53 immunoreactivity were significantly associated with recurrence $(\mathrm{p}<$ .01). Invasion with extensive p53 immunoreactivity, and invasion with increased mitotic activity, were not significantly associated with recurrence, with the exception of combinations with specific two features, $\mathrm{Ki}-67 \mathrm{LI}>3 \%$ with invasion and $\mathrm{Ki}-67 \mathrm{LI}>3 \%$ with increased mitotic activity. Although there was a statistical association with recurrence in the group with extensive p53 immunoreactivity, and increased mitotic activity $(\mathrm{p}=.03)$, a careful interpretation is needed because there was only one case which met this criteria. Although it can be assumed that the statistical significance of $\mathrm{Ki}-67 \mathrm{LI}>3 \%$ affected the result to some degree, pairing $\mathrm{Ki}-67 \mathrm{LI}>3 \%$ with one of the other factors, except extensive p53 immunoreactivity, was not associated with significant differences.

The mean age of the group with recurrence was significantly younger than the other group and Matsuyama ${ }^{21}$ found similar results. Comparison of PA and aPA indicated that the result from Chiloiro et al. ${ }^{14}$ was significant, however that from Zada et al. ${ }^{9}$ was not. Chiloiro et al. ${ }^{14}$ and Zada et al. ${ }^{9}$ both used the criteria without invasion.

Using linear by linear association, we found that larger tumors recurred more frequently; however, this result was not statistically significant. In comparison, giant adenomas were significantly associated with recurrence. However, Matsuyama ${ }^{21}$ reported that there was no significant relationship between recurrence and the giant tumors, but Lee et al. ${ }^{10}$ reported that tumors which measured $\geq 25 \mathrm{~mm}$ significantly recurred more frequently than tumors that were $<25 \mathrm{~mm}$. Tortosa and $\mathrm{Webb}^{13}$ found that tumors that were $\geq 1 \mathrm{~cm}$ were identified significantly more frequently in aPA than PA. In spite of the same cut-off for size as Tortosa and Webb, ${ }^{13}$ Zada et al. ${ }^{9}$ and Chiloiro et al. ${ }^{14}$ found no significant differences.

The group with residual masses after surgery showed significantly more recurrence and Matsuyama ${ }^{21}$ reported similar results. However, these results were different from those reported by Lee et al. ${ }^{10}$ According to Chiloiro et al. ${ }^{14}$, there was no significant difference between PA and aPA. Because residual mass assessment was performed by gross examination at surgery or radiologic methods with controversial criteria, differentiating residual mass after excision and true tumor recurrence is limited.

There were some limitations to this study: (1) the number of cases retrieved was relatively small, (2) the possibility of radiologic inaccuracy in the assessment of invasion or residual mass after surgery, and (3) no definite standard for extensive p53 immunoreactivity and increased mitotic activity, such as cut-off or intensity. We investigated the predictive value of clinical and pathologic characteristics in relation to recurrence. Even though PA cases did not meet all the criteria, using any single pathologic feature can be useful to predict recurrence. Ki-67 LI with a cut-off $>3 \%$ with or without extensive p53 immunoreactivity ( $\geq 20 \%)$ was significantly associated with recurrence. Assessment of these features can be applied in PA diagnosis for more accurate prediction of recurrence. Additional studies with a larger sample population will be important for identifying more detailed, reproducible and clear features for markers that can be used to predict recurrence.

\section{Conflicts of Interest}

No potential conflict of interest relevant to this article was reported.

\section{Acknowledgments}

We would like to sincerely thank Yo-Sep Chong for his devoted efforts in editing and reviewing this article.

\section{REFERENCES}

1. DeLellis RA, Lloyd RV, Heitz PU, Eng C. World Health Organization classification of tumours: pathology and genetics of tumours 
of endocrine organs. Lyon: IARC Press, 2004.

2. Kaltsas GA, Nomikos P, Kontogeorgos G, Buchfelder M, Grossman AB. Clinical review: diagnosis and management of pituitary carcinomas. J Clin Endocrinol Metab 2005; 90: 3089-99.

3. Fernandez A, Karavitaki N, Wass JA. Prevalence of pituitary adenomas: a community-based, cross-sectional study in Banbury (Oxfordshire, UK). Clin Endocrinol (Oxf) 2010; 72: 377-82.

4. Daly AF, Rixhon M, Adam C, Dempegioti A, Tichomirowa MA, Beckers A. High prevalence of pituitary adenomas: a cross-sectional study in the province of Liege, Belgium. J Clin Endocrinol Metab 2006; 91: 4769-75.

5. Saeger W, Ludecke DK, Buchfelder M, Fahlbusch R, Quabbe HJ, Petersenn S. Pathohistological classification of pituitary tumors: 10 years of experience with the German Pituitary Tumor Registry. Eur J Endocrinol 2007; 156: 203-16.

6. Al-Shraim M, Asa SL. The 2004 World Health Organization classification of pituitary tumors: what is new? Acta Neuropathol 2006; 111: $1-7$.

7. Mete O, Ezzat S, Asa SL. Biomarkers of aggressive pituitary adenomas. J Mol Endocrinol 2012; 49: R69-78.

8. Yildirim AE, Divanlioglu D, Nacar OA, et al. Incidence, hormonal distribution and postoperative follow up of atypical pituitary adenomas. Turk Neurosurg 2013; 23: 226-31.

9. Zada G, Woodmansee WW, Ramkissoon S, Amadio J, Nose V, Laws ER Jr. Atypical pituitary adenomas: incidence, clinical characteristics, and implications. J Neurosurg 2011; 114: 336-44.

10. Lee EH, Kim KH, Kwon JH, Kim HD, Kim YZ. Results of immunohistochemical staining of cell-cycle regulators: the prediction of recurrence of functioning pituitary adenoma. World Neurosurg 2014; 81: 563-75.

11. Sadeghipour A, Mahouzi L, Salem MM, et al. Ki67 labeling correlated with invasion but not with recurrence. Appl Immunohistochem Mol Morphol 2016 Feb 9 [Epub]. https://doi.org/10.1097/

\section{PAI.0000000000000303.}

12. Hadzhiyanev A, Ivanova R, Nachev E, et al. Evaluation of prognostic utility of MIB-1 and p53 expression in pituitary adenomas: correlations with clinical behaviour and follow-up results. Biotechnol Biotechnol Equip 2014; 28: 502-7.

13. Tortosa F, Webb SM. Atypical pituitary adenomas: 10 years of experience in a reference centre in Portugal. Neurologia 2016; 31: 97-105.

14. Chiloiro S, Doglietto F, Trapasso B, et al. Typical and atypical pituitary adenomas: a single-center analysis of outcome and prognosis. Neuroendocrinology 2015; 101: 143-50.

15. Szumilas M. Explaining odds ratios. J Can Acad Child Adolesc Psychiatry 2010; 19: 227-9.

16. Figarella-Branger D, Trouillas J. The new WHO classification of human pituitary tumors: comments. Acta Neuropathol 2006; 111: 71-2.

17. Grossman AB. The 2004 World Health Organization classification of pituitary tumors: is it clinically helpful? Acta Neuropathol 2006; 111: 76-7.

18. Kleinschmidt-DeMasters BK. Subtyping does matter in pituitary adenomas. Acta Neuropathol 2006; 111: 84-5.

19. Laws ER Jr, Lopes MB. The new WHO classification of pituitary tumors: highlights and areas of controversy. Acta Neuropathol 2006; 111: 80-1.

20. Maclean J, Aldridge M, Bomanji J, Short S, Fersht N. Peptide receptor radionuclide therapy for aggressive atypical pituitary adeno$\mathrm{ma} /$ carcinoma: variable clinical response in preliminary evaluation. Pituitary 2014; 17: 530-8.

21. Matsuyama J. Ki-67 expression for predicting progression of postoperative residual pituitary adenomas: correlations with clinical variables. Neurol Med Chir (Tokyo) 2012; 52: 563-9.

22. Miermeister CP, Petersenn S, Buchfelder M, et al. Histological criteria for atypical pituitary adenomas: data from the German pituitary adenoma registry suggests modifications. Acta Neuropathol Commun 2015; 3: 50. 